\title{
Difficult airway management in the neonate; benchmarking simulation trainers
}

Authors:

Damien M. Gilby FRACP ${ }^{1,3}$, Noel Roberts FANZCA ${ }^{2}$, Alice Stewart FRACP ${ }^{1}$, Risha Bhatia PhD ${ }^{1,3}$, Calum Roberts $\mathrm{PhD}^{1,3}$

1. Monash Newborn, Monash Children's Hospital, Clayton, Melbourne, Australia.

2. Department of Anaesthetics, Monash Medical Centre, Clayton, Melbourne, Australia.

3. Department of Paediatrics, Monash University, Melbourne, Australia

\section{Background and Aim:}

To evaluate the suitability of two advanced simulation manikins for training in the management of the difficult infant and neonatal airway.

\section{Methods:}

Neonatologists, anaesthetists, paediatric intensivists and senior trainees experienced in airway management (mean specialist experience 12 years) performed a structured assessment on three manikins: the AirSim ${ }^{\circledR}$ Pierre Robin and the Life/form ${ }^{\circledR}$ C.H.A.R.L.I.E. as simulators of a difficult neonatal airway, and the SimNewB $\circledast$, a standard neonatal airway manikin ${ }^{1-3}$. Procedures performed on each manikin included mask ventilation, laryngeal mask insertion, direct laryngoscopic intubation and indirect video laryngoscopy with the GlideScope ${ }^{\circledR}$ Advanced Video Laryngoscope. The fidelity of each manikin was assessed using Likert scale questionnaires and objective measures of procedural success.

Table 1: Procedure first attempt success and time to intubation by manikin. Success reported as $n(\%)$, duration as median (range)

\begin{tabular}{|l|c|c|c|}
\hline & SimNewB $^{\circledR}$ & $\begin{array}{c}\text { AirSim } \\
\text { Pierre Robin }^{\circledR}\end{array}$ & $\begin{array}{c}\text { Life/form } \\
\text { C.H.A.R.L.I.E. }\end{array}$ \\
\hline $\begin{array}{l}\text { Miller blade first attempt } \\
\text { success n(\%) }\end{array}$ & $21(95)$ & $20(91)$ & $20(91)$ \\
\hline $\begin{array}{l}\text { Miller blade } \\
\text { median(range) first } \\
\text { attempt duration (s) }\end{array}$ & $17(8-42)$ & $19(11-69)$ & $25(15-50)^{\star}$ \\
\hline $\begin{array}{l}\text { GVL first attempt success } \\
\text { n(\%) }\end{array}$ & $20(91)$ & $22(100)$ & $20(91)$ \\
\hline $\begin{array}{l}\text { GVL median(range) first } \\
\text { attempt duration (s) }\end{array}$ & $18(13-82)$ & $33(13-106)$ & $55(18-110) \dagger$ \\
\hline $\begin{array}{l}\text { Laryngeal mask insertion } \\
\text { and ventilation first } \\
\text { attempt success n(\%) }\end{array}$ & $17(77)$ & $1(5) f$ & $21(95)$ \\
\hline $\begin{array}{l}\text { Mask ventilation first } \\
\text { attempt success n(\%) }\end{array}$ & $19(86)$ & $22(100)$ & $21(95)$ \\
\hline
\end{tabular}

$\mathrm{GVL}=$ GlideScope ${ }^{\circledR}$ Advanced Video Laryngoscope

${ }^{\star} \mathrm{P}=0.006$ In comparison with SimNewB ${ }^{\circledR}$ (Kruskal-Wallis test with Dunn's multiple comparisons test)

$\dagger P=0.043$ In comparison with SimNewB ${ }^{\circledR}$ (Kruskal-Wallis test with Dunn's multiple comparisons test)

$f \mathrm{P}<0.001$ In comparison with $\operatorname{SimNewB}{ }^{\circledR}$ and C.H.A.R.L.I.E. (Fisher's exact test)

\section{References:}

1. LTD T. AirSim Pierre Robin Manual Belfast, Northern Ireland [accessed 28/08/2017]. Available from:

http://trucorp.com/uploads/manuals/airsim-pierre-robin.pdf.

2. Healthcare N. Life/Form ${ }^{\circledR}$ Nasco Healthcare C.H.A.R.L.I.E. Neonatal

Resuscitation Simulator Manual: Nasco Healthcare, Fort Atkinson, WI, USA; [Available from:

\section{Results:}

A total of 22 expert clinicians completed 156 procedures and 66 manikin evaluations. Despite differences in individual task performances and fidelity scores, participants either somewhat or strongly agreed that the AirSim $®$ and Life/form $®$ were realistic representations of a difficult airway (64\% and $80 \%$ respectively), and should be used for difficult airway training (65\% and $75 \%$ ). Intubation first attempt success did not differ between manikins, but duration of intubation attempt was increased with the Life/ form $®$ (Table 1). Proportion of intubation success over time is shown in Figures 1 and 2. Successful laryngeal mask ventilation of the AirSim® was achieved by just one participant.

\section{Conclusion:}

Clinicians should be aware of key differences between these manikins when considering purchase, planning a simulation program, or using the manikins to test equipment.

Figure 1: Kaplan Meier curve: proportion intubated successfully over time using Miller blade

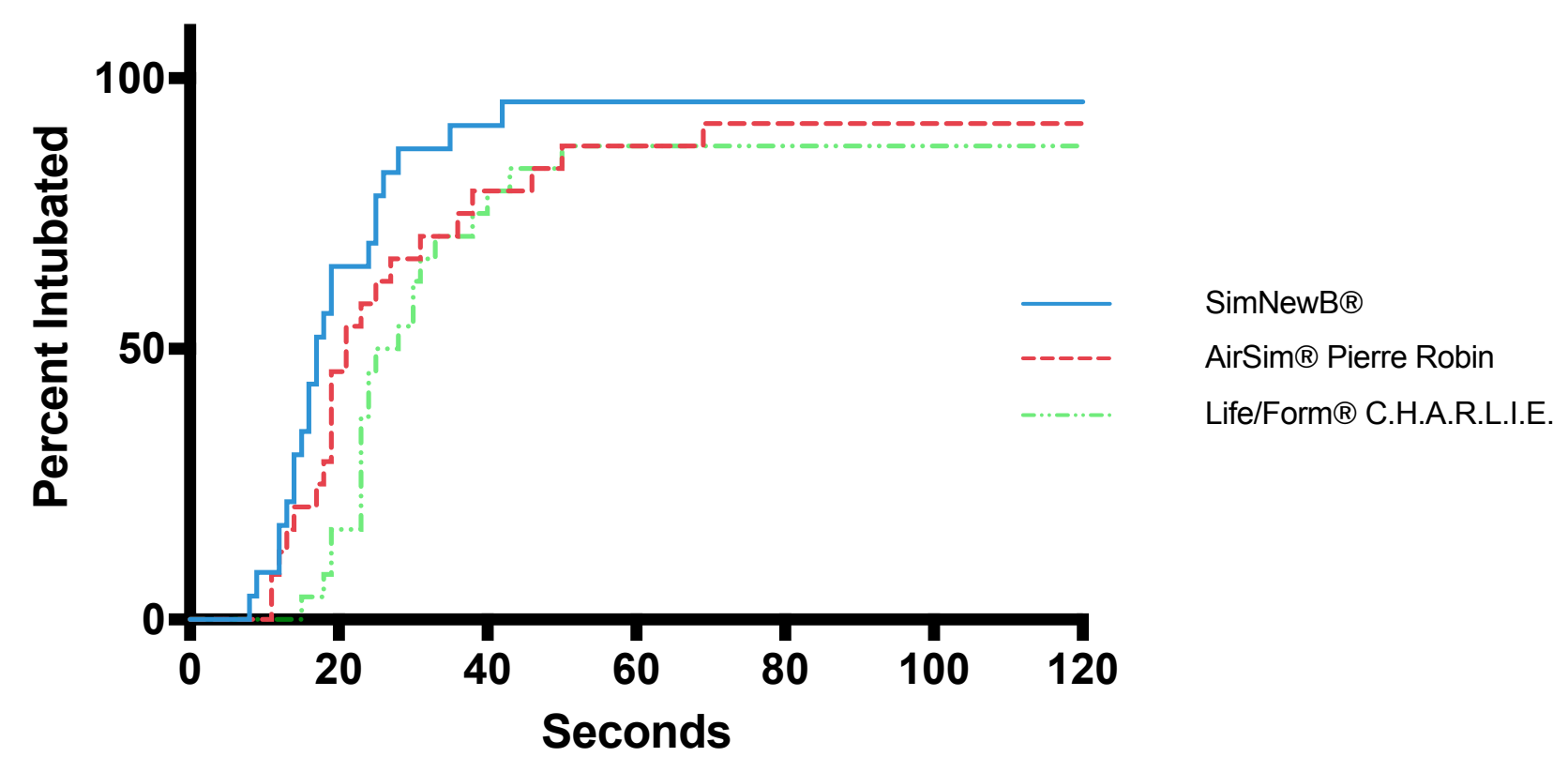

Adjusted $P=0.02$ comparing C.H.A.R.L.I.E. curve with SimNewB ${ }^{\circledR}$; Mantel-Cox test with Bonferroni post test correction

Figure 2: Kaplan Meier curve: proportion intubated successfully over time using GlideScope ${ }^{\circledR}$ Advanced Video Laryngoscope.

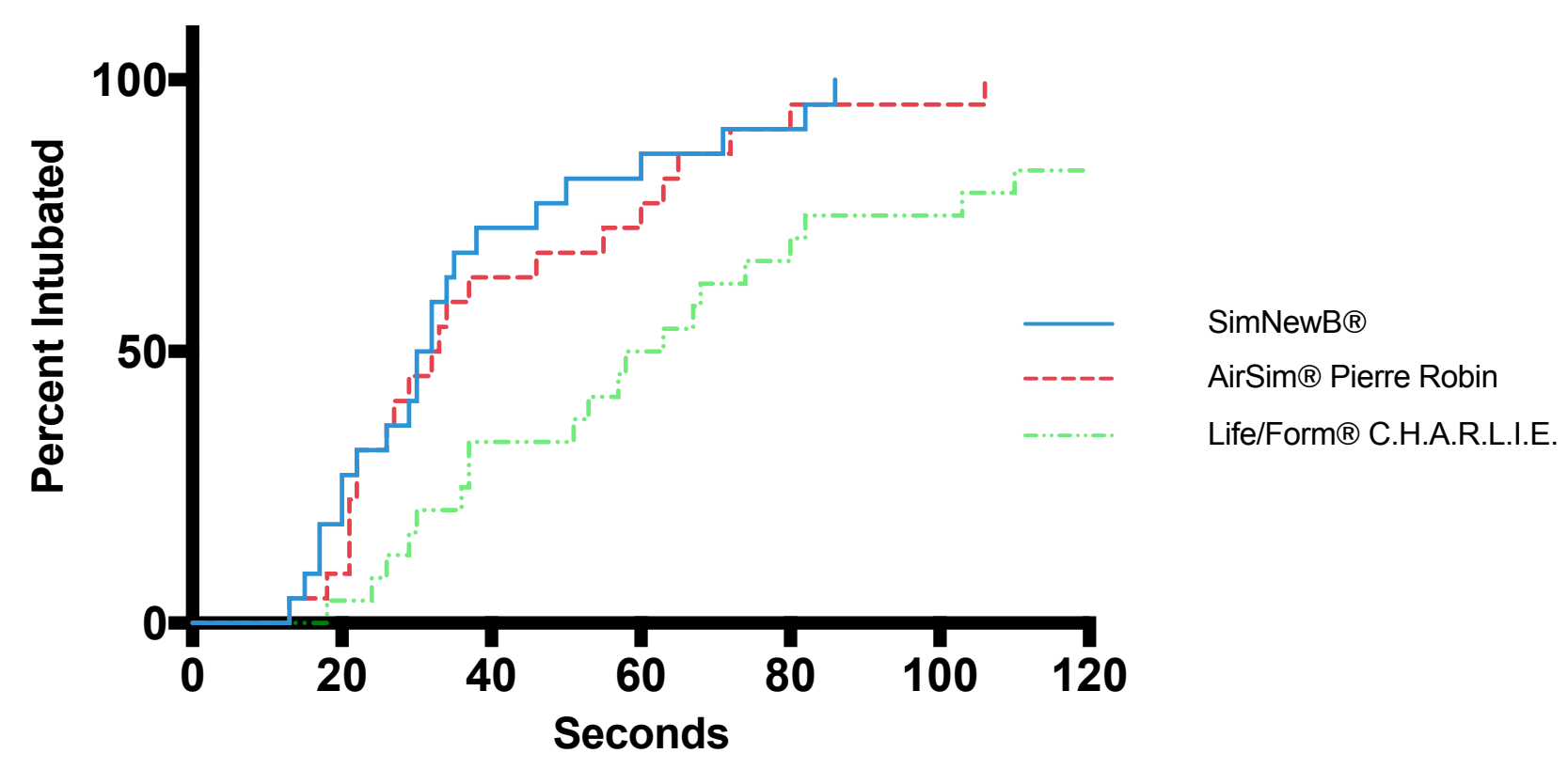

Adjusted $P=0.005$ comparing C.H.A.R.L.I.E. curve with SimNewB ${ }^{\circledR}, P=0.02$ comparing C.H.A.R.L.I.E. with AirSim Pierre Robin; Mantel-Cox test with Bonferroni post test correction 